\title{
Differential Expression of Glut1 in Pulmonary Neuroendocrine Tumors: Correlation with Histological Grade
}

Hyun Ju Lee ${ }^{1} \cdot$ Seol Bong Yoo ${ }^{1}$ Won Woo Lee ${ }^{2} \cdot$ Doo Hyun Chung Jeong-Wook Seo ${ }^{1}$ Jin-Haeng Chung ${ }^{1,3}$

Departments of ${ }^{1}$ Pathology and ${ }^{2}$ Nuclear Medicine, Seoul National University College of Medicine, Seoul; ${ }^{3}$ Respiratory Center, Seoul National University Bundang Hospital, Seongnam, Korea

Received : December 8, 2008 Accepted : February 5, 2009

\section{Corresponding Author}

Jin-Haeng Chung, M.D.

Department of Pathology, Seoul National University

College of Medicine, Seoul National University

Bundang Hospital, 16-6 Gumi-ro, Bundang-gu,

Seongnam 463-707, Korea

Tel: 031-787-7713

Fax: 031-787-4012

E-mail: chungih@snu.ac.kr

*This work was supported by grant no 02-2008-029 from the SNUBH Research Fund and partly supported by the Korean Science \& Engineering Foundation (KOSEF) through the Tumor Immunity Medical

Research Center at Seoul National University College of Medicine.
Background : Increased glucose uptake, a process that is mediated by glucose transporter (Glut1) proteins, is an important metabolic feature in a variety of cancer cells. The overexpression of Glut1 in human cancers is known to be related to a variety of histopathological parameters, including histological grade, proliferation rate, and lymphatic invasion. The principal objective of this study was to evaluate Glut1 expression in the spectrum of pulmonary neuroendocrine (NE) tumors including typical carcinoid tumor (TC), atypical carcinoid tumor (AC), large cell neuroendocrine carcinoma (LCNEC), and small cell carcinoma (SCC), and to characterize the relationship between Glut1 expression and the histologic grade of NE tumors. Methods : 19 TC, 7 AC, 13 LCNEC, and 6 SCC patients were included in this study. The percentages of Glut1-positive tumor cells in these patients were determined. For statistical analysis, Glut1 expression was subdivided into a Glut1-low expression group (0 -30\%) and a Glut1high expression group (31-90\%). Results : In our subgroup analyses, the histological grade of pulmonary neuroendocrine (NE) tumors was significantly correlated with Glut1 expression; TC $(n=19,3.6 \pm 4.2 \%), A C(n=7,20.0 \pm 4.9 \%)$, LCNEC $(n=13,60.0 \pm 21.1 \%)$, and SCC $(n=$ $6,74.2 \pm 16.9 \%$ ). Glut1-high expression was significantly associated with high-grade NE tumors such as LCNEC and SCC $(n=19,62.6 \pm 21.0 \%)(p=0.000)$. Conclusions : The results of this study appear to indicate that Glut1 overexpression is a consistent feature of high-grade NE lung tumors.

Key Words : GLUT1 Protein; Glucose transporter; Neuroendocrine tumors; Lung neoplasms; Immunohistochemistry
In the recent revision of the $2004 \mathrm{WHO}$ classification of lung and pleural tumors, ${ }^{1}$ pulmonary neuroendocrine (NE) tumors include typical carcinoid (TC), a low-grade malignancy; atypical carcinoid (AC), an intermediate -grade malignancy; and large cell neuroendocrine carcinoma (LCNEC) and small cell carcinoma (SCC), both of which are high-grade malignancies. The prognosis and biologic behaviors of NE tumors deteriorate according to their histologic grade. ${ }^{2}$ Treatment depends heavily on the histologic features, which reflect differences in clinical behavior and prognosis.

Glut1 is a facilitative glucose transporter transmembrane protein, which is physiologically expressed and immunohistochem- ically detectable in red blood cell membranes, ${ }^{3}$ brain capillary endothelium (the blood brain barrier), ${ }^{4}$ and the perineurium of the peripheral nerve. It is also expressed in the placenta ${ }^{5}$ basal cells of the benign squamous epithelium, ${ }^{6}$ reactive epidermal cells, and many epithelial neoplasms. It plays a mediating role in cellular glucose uptake. Malignant cells evidence increased glucose uptake and utilization when compared to their benign/ normal counterparts in vitro and in vitro. ${ }^{7}$ Several studies have reported an association between Glut 1 expression and neoplastic progression in various human cancers, including ovarian epithelial malignancy, ${ }^{8}$ colorectal adenocarcinoma, ${ }^{9}$ adenocarcinoma arising in Barrett's metaplasia of the esophagus, ${ }^{10}$ endome- 
trial adenocarcinomas, ${ }^{11}$ human head and neck tumors, ${ }^{12}$ breast cancer $^{13}$ and renal cell carcinomas. ${ }^{14}$

Song et al. ${ }^{15}$ previously reported that Glutl expression in pulmonary NE tumors was correlated with 18F-fluorodeoxyglucose (FDG) uptake on positron emission tomography (PET). However, the majority of the specimens included in the previous study were obtained from small biopsy samples, and the correlation of Glut1 expression with histologic grade has yet to be fully evaluated. Thus, we enrolled only surgically resected NE tumors and investigated Glut1 expression in terms of histologic grade of malignancy.

\section{MATERIALS AND METHODS}

\section{Patients}

Forty-five patients with NE lung tumors (35 men, 10 women: age range 24-80 year; mean 58 year) who underwent curative surgical resection at the Seoul National University Hospital and
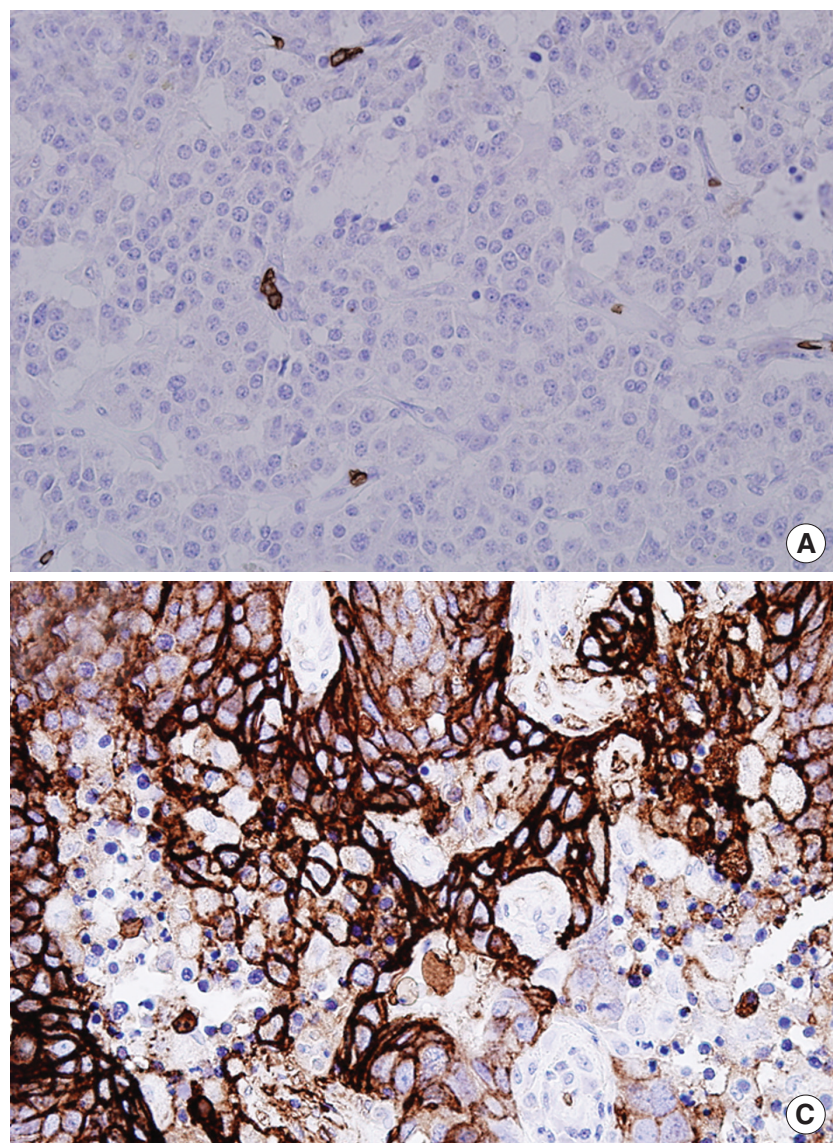

Bundang Hospital between 1995 and 2006 were enrolled in this study. H\&E-stained slides of each tumor were reviewed for subtype in the NE lung tumors, and the tumors were then reclassified in accordance with the 2004 WHO classification system. The pathologic diagnoses were as follows: TC $(n=19)$, AC $(n=7)$, $\operatorname{LCNEC}(n=13)$, and SCC ( $n=6)$.

Immunohistochemistry

Formalin-fixed, paraffin-embedded $4 \mu \mathrm{m}$ tissue sections were immunostained with rabbit anti-Glut1 polyclonal antibody for Glut1 (1:50, Neomarkers, Fremont, CA, USA), via a previously described procedure. ${ }^{15,16}$ Adjacent sections incubated with rabbit IgG were employed as negative controls. Red blood cells present in each section were used as positive controls for Glut1. Glut1 expression was considered positive only if distinct membrane staining was detected. Glut1 expression was presented as $\%$ Glut1 expression, and the percentages of immunostainingpositive cells were measured by counting tumor cell numbers, i.e. the number of distinctly stained membranes per 1,000 tumor
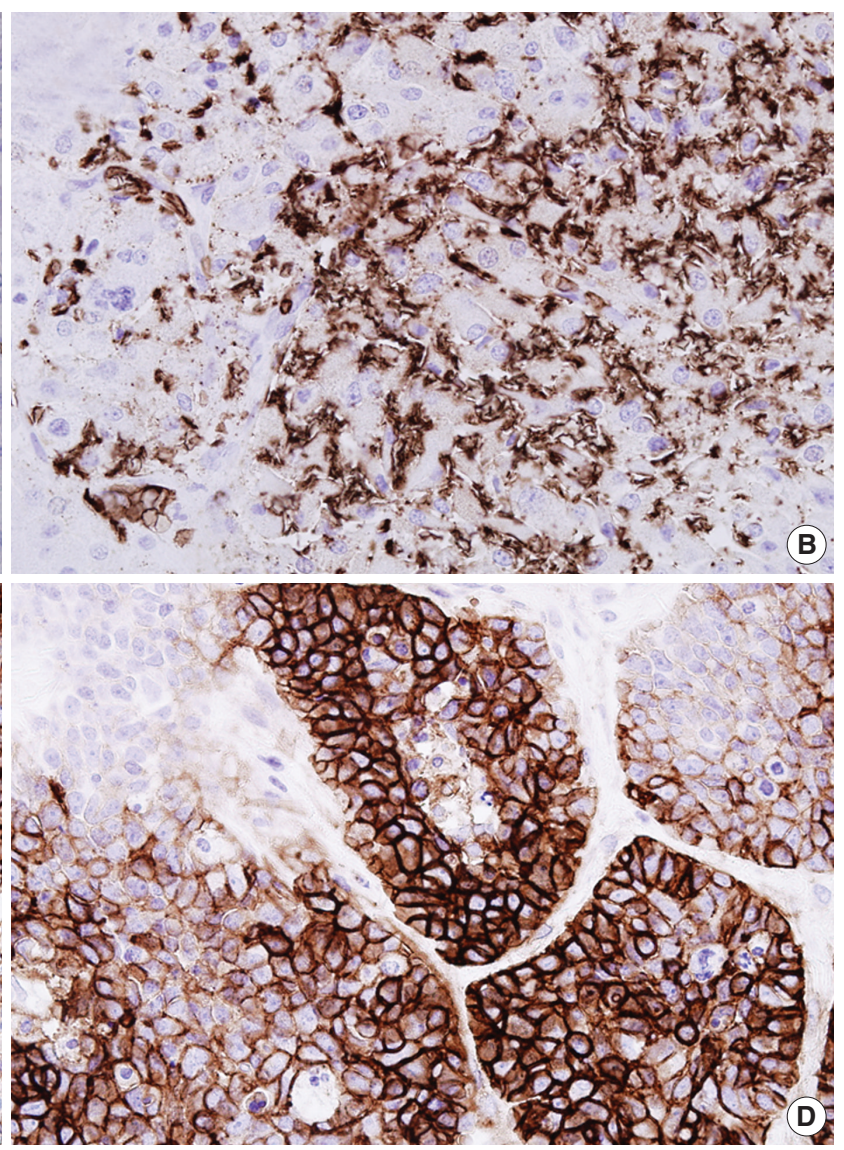

Fig. 1. Immunohistochemical staining of Glut1 expression in NE tumors of the lung: TC (A), AC (B) , LCNEC (C) and SCC (D). Glut1 was expressed in the membrane of tumor cells $(\times 400)$. 
cells in the most representative region at 400X magnification.

\section{Statistical analysis}

The analyses in this study were conducted using the SPSS software package for Windows, version 12.0. The relationship between \%Glut1 expression and histological grade was assessed via Pearson Chi-Square analysis. p-values $<0.05$ were considered statistically significant.

\section{RESULTS}

The Glut1 staining was detected primarily in the membranous pattern (Fig. 1). A case of TC showed diffuse intracytoplasmic staining without a membranous pattern, and was considered $0 \%$. The proportion of Glut1 expression in all NE tumors varied in accordance with histologic type. The \% Glut1 expression of NE lung tumors ranged between 0 and $90 \%$ (mean $30.8 \pm$ $31.2 \%$ ), and the means of \%Glut 1 expressions of TC, AC, LCNEC, and SCC were $3.6 \pm 4.2 \%, 20.0 \pm 4.9 \%, 60.0 \pm 21.1 \%$, and $74.2 \pm 16.9 \%$, respectively (Fig. 2).

For statistical analysis, the cases were subdivided into a Glut1low expression group (0-30\%) and a Glut1-high expression group (30-90\%). NE lung tumors were also subdivided into lowto-intermediate grade and high-grade. Glut1-high expression was significantly associated with high-grade NE lung tumors $(\mathrm{p}=0.000)$ (Table 1).

\section{DISCUSSION}

Glut1, the human erythrocyte glucose transporter, is a member of an expanding family of transmembrane proteins known

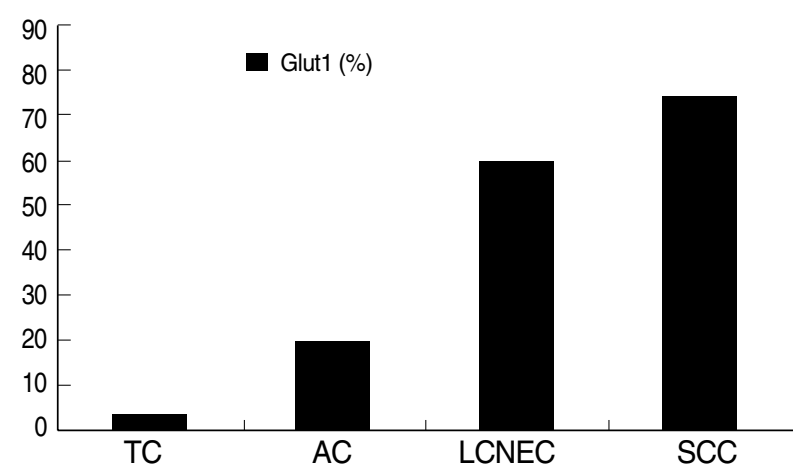

Fig. 2. Glut1 immunoreactivity in histologic types of neuroendocrine tumors of the lung. as the facilitative glucose transporters, a family which currently comprises 12 members. ${ }^{3}$ Under physiological conditions, Glut1 is expressed in a limited number of organs, including the brain, placenta, breast, and kidney, in addition to $\mathrm{RBC} \cdot{ }^{17}$ Recently, Glut1 has become the focus of considerable attention with FDGPET in tumor biology. ${ }^{15,16,18,19}$ Glut1 expression has been noted in a broad variety of human tumors, including ovarian epithelial malignancy, ${ }^{8}$ adenocarcinomas of endometrial ${ }^{11}$ and colonic origin, ${ }^{9}$ invasive and in situ squamous carcinomas of the skin, ${ }^{20}$ head and neck squamous carcinomas, ${ }^{12}$ papillary thyroid carcinoma ${ }^{21}$ renal cell carcinoma ${ }^{14}$ esophageal Barrett-associated adenocarcinoma, ${ }^{10}$ nonsmall cell lung carcinoma ${ }^{16,22-25}$ and breast carcinomas. ${ }^{26}$ Additionally, Glut1 is associated with significantly more aggressive tumor course, thus indicating that Glut1, a highly efficient glucose transporter, may perform a role in maintaining the high-energy requirements of aggressive carcinomas. Thus, Glut1 expression has been identified as a possible new diagnostic and prognostic marker in certain human cancers. ${ }^{8,911,22,26}$

In this study, we assessed Glut1 expression in NE lung tumors via immunohistochemistry. We determined that high Glut1 expression levels in NE lung tumors were associated with highgrade histology. This is why the criteria for NE lung tumor histologic grade include mitosis and tumor proliferative activity. Glut1 expression has been associated with histologic subtype, lymphatic invasion, lymph node metastasis, Ki-67 proliferative index, and so forth. ${ }^{916,19}$ However, the principal criterion for the histologic grade of squamous cell carcinoma is morphological squamous differentiation (well, moderate, poorly differentiated), such that high Glut1 expression is unrelated to high-grade histology. ${ }^{16,23-25}$ Low expression of Glut1 staining in TC, in contrast to the middling levels of expression seen in intermediate grade $\mathrm{AC}$ tumors and the high expression in high-grade $\mathrm{NE}$ tumors such as LCNEC and SCC, is consistent with findings in several other anatomic sites. ${ }^{8,9,11}$

Table 1. Correlations between low or high Glut1 expression and histologic grade of neuroendocrine tumors of the lung

\begin{tabular}{|c|c|c|c|}
\hline & $\begin{array}{l}\text { Low to interme- } \\
\text { diate grade }\end{array}$ & High grade & \multirow{2}{*}{$\begin{array}{c}\text { Total } \\
(n=45)\end{array}$} \\
\hline & $\begin{array}{c}\text { TC and } \\
\text { AC }(n=26)\end{array}$ & $\begin{array}{l}\text { LCNEC and } \\
\text { SCC }(n=19)\end{array}$ & \\
\hline Glut1-low & 26 & 2 & 28 \\
\hline Glut1-high & 0 & 17 & 17 \\
\hline p-value & $<0.001$ & $<0.001$ & \\
\hline Mean of \%Glut1 (\%) & $7.5 \pm 7.7$ & $62.6 \pm 21.0$ & $30.8 \pm 31.2$ \\
\hline
\end{tabular}

TC, typical carcinoid; AC, atypical carcinoid; LCNEC, large cell neuroendocrine carcinoma; SCC, small cell lung carcinoma. 
According to Song et al., ${ }^{15}$ Glut 1 expression in NE lung tumors is correlated positively with FDG uptake. The authors considered all kinds of Glut1 expression patterns, i.e., intracytoplasmic, membranous or mixed intracytoplasmic and membranous patterns as a positive reaction, in contrast to other papers concerning Glut1 immunohistochemical staining. In this paper, a very interesting TC case was reported with high FDG uptake and diffuse intracytoplasmic Glut1 expression, rather than a membranous pattern. As Glut1 is a transmembrane protein, when evaluating Glut1 expression, the membranous pattern should be considered, as was the case in other papers. ${ }^{8,11,16} \mathrm{We}$ concluded that a membranous pattern is the only reliable criterion for true expression of Glut1 as a transporter protein, and regarded the Glut 1 expression of TC as $0 \%$. In that case, the patient had been regularly attending a hospital for treatment of Cushing's syndrome, and he was already aware of the existence of a solitary mass in the lung, and also that the size of the mass had remained constant for 20 years. The clinicians in this case suspected that the solitary mass was a malignancy, based on the high uptake noted upon FDG-PET examination (max SUV 29.5). After surgery, it was histologically confirmed as a TC and the patient's condition evidenced benign biologic behavior, without recurrence or metastasis for 4 years. This case may represent an extreme example of TC. The diffuse intracytoplasmic pattern of Glut 1 expression evidenced by the TC might be explained as follows: 1) anti-Glut1 antibody was stained nonspecifically in the intracytoplasmic NE secretory granules, 2) glucose was not catalytically hydrolyzed by glucose-6-phosphatase, and intracytoplasmic accumulation occurred via some abnormal mechanism. Thus, we should not oversimplify the case simply because the TC evidenced abundant expression of Glut 1. Further large-scale studies are recommended to generate more information regarding Glut1 metabolism in NE lung tumors.

In summary, we determined that Glut1 overexpression is a consistent feature of high-grade NE lung tumors. Although the biological function of Glut1 in NE lung tumors remains to be thoroughly explained, Glut1 expression should be considered a possible source of new prognostic information about histologic grade.

\section{REFERENCES}

1. Travis WD, Brambilla E, Muller-Hermelink HK, Harris CC. World Health Organization International Histological Classification of Tumours. Pathology and genetics of tumors of the lung, pleura, thymus and heart. Lyon: IARC Press, 2004.

2. Travis WD, Gal AA, Colby TV, Klimstra DS, Falk R, Koss MN. Reproducibility of neuroendocrine lung tumor classification. Hum Pathol 1998; 29: 272-9.

3. Pessin JE, Bell GI. Mammalian facilitative glucose transporter family: structure and molecular regulation. Annu Rev Physiol 1992; 54 911-30.

4. Pardridge WM, Boado RJ, Farrell CR. Brain-type glucose transporter (GLUT-1) is selectively localized to the blood-brain barrier: studies with quantitative western blotting and in situ hybridization. J Biol Chem 1990; 265: 18035-40.

5. Takata K, Kasahara T, Kasahara M, Ezaki O, Hirano H. Localization of erythrocyte/HepG2-type glucose transporter (GLUT1) in human placental villi. Cell Tissue Res 1992; 267: 407-12.

6. Voldstedlund M, Dabelsteen E. Expression of GLUT1 in stratified squamous epithelia and oral carcinoma from humans and rats. APMIS 1997; 105: 537-45.

7. Isselbacher KJ. Sugar and amino acid transport by cells in culture: differences between normal and malignant cells. N Engl J Med 1972; 286: 929-33.

8. Kalir T, Wang BY, Goldfischer M, et al. Immunohistochemical staining of GLUT1 in benign, borderline, and malignant ovarian epithelia. Cancer 2002; 94: 1078-82.

9. Younes M, Lechago LV, Lechago J. Overexpression of the human erythrocyte glucose transporter occurs as a late event in human colorectal carcinogenesis and is associated with an increased incidence of lymph node metastases. Clin Cancer Res 1996; 2: 1151-4.

10. Younes M, Ertan A, Lechago LV, Somoano J, Lechago J. Human erythrocyte glucose transporter (Glut1) is immunohistochemically detected as a late event during malignant progression in Barrett's metaplasia. Cancer Epidemiol Biomarkers Prev 1997; 6: 303-5.

11. Wang BY, Kalir T, Sabo E, Sherman DE, Cohen C, Burstein DE. Immunohistochemical staining of GLUT1 in benign, hyperplastic, and malignant endometrial epithelia. Cancer 2000; 88: 2774-81.

12. Mellanen P, Minn H, Grenman R, Harkonen P. Expression of glucose transporters in head-and-neck tumors. Int J Cancer 1994; 56: $622-9$.

13. Brown RS, Wahl RL. Overexpression of Glut-1 glucose transporter in human breast cancer: an immunohistochemical study. Cancer 1993; 72: 2979-85.

14. Nagase Y, Takata K, Moriyama N, Aso Y, Murakami T, Hirano H. Immunohistochemical localization of glucose transporters in human renal cell carcinoma. J Urol 1995; 153: 798-801.

15. Song YS, Lee WW, Chung JH, Park SY, Kim YK, Kim SE. Correlation between FDG uptake and glucose transporter type 1 expression in neuroendocrine tumors of the lung. Lung Cancer 2008; 61: 54-60. 
16. Chung JH, Cho KJ, Lee SS, et al. Overexpression of Glut1 in lymphoid follicles correlates with false-positive (18)F-FDG PET results in lung cancer staging. J Nucl Med 2004; 45: 999-1003.

17. Younes M, Lechago LV, Somoano JR, Mosharaf M, Lechago J. Wide expression of the human erythrocyte glucose transporter Glut1 in human cancers. Cancer Res 1996; 56: 1164-7.

18. Khandani AH, Whitney KD, Keller SM, Isasi CR, Donald Blaufox M. Sensitivity of FDG PET, GLUT1 expression and proliferative index in bronchioloalveolar lung cancer. Nucl Med Commun 2007; 28: 173-7.

19. Nguyen XC, Lee WW, Chung JH, et al. FDG uptake, glucose transporter type 1, and Ki-67 expressions in non-small-cell lung cancer: correlations and prognostic values. Eur J Radiol 2007; 62: 214-9.

20. Baer SC, Casaubon L, Younes M. Expression of the human erythrocyte glucose transporter Glut1 in cutaneous neoplasia. J Am Acad Dermatol 1997; 37: 575-7.

21. Haber RS, Weiser KR, Pritsker A, Reder I, Burstein DE. GLUT1 glucose transporter expression in benign and malignant thyroid nod- ules. Thyroid 1997; 7:363-7.

22. Younes M, Brown RW, Stephenson M, Gondo M, Cagle PT. Overexpression of Glut1 and Glut3 in stage I nonsmall cell lung carcinoma is associated with poor survival. Cancer 1997; 80: 1046-51.

23. Brown RS, Leung JY, Kison PV, Zasadny KR, Flint A, Wahl RL. Glucose transporters and FDG uptake in untreated primary human nonsmall cell lung cancer. J Nucl Med 1999; 40: 556-65.

24. Ito T, Noguchi Y, Satoh S, Hayashi H, Inayama Y, Kitamura H. Expression of facilitative glucose transporter isoforms in lung carcinomas: its relation to histologic type, differentiation grade, and tumor stage. Mod Pathol 1998; 11: 437-43.

25. Wong CY, Nunez R, Bohdiewicz P, et al. Patterns of abnormal FDG uptake by various histological types of non-small cell lung cancer at initial staging by PET. Eur J Nucl Med 2001; 28: 1702-5.

26. Younes M, Brown RW, Mody DR, Fernandez L, Laucirica R. GLUT1 expression in human breast carcinoma: correlation with known prognostic markers. Anticancer Res 1995; 15: 2895-8. 